\title{
Sonographic Characterisation of Tissue Changes Associated with Infused Apomorphine Hydrochloride: A Case Series
}

Hazel Edwards, Leon Poltawski \& Amy Todd

School of Health \& Emergency Professions, University of Hertfordshire, UK

\begin{abstract}
Apomorphine hydrochloride is used to treat refractory motor fluctuations in advanced Parkinson's disease. Delivery by infusion is associated with the formation of hard subcutaneous nodules, which may be painful, limit available infusion sites, and interfere with absorption of the drug. Diagnostic ultrasound was used to image affected tissue in 12 people experiencing apomorphine nodule formations. It revealed significant departures from normality in dermal and subcutaneous tissue, including a variety of nodule appearances, dermal thickening and diffuse changes consistent with inflammatory reactions. Sonographic appearances differed markedly between individuals, but no clear correlation between severity of tissue changes, patient demographics and apomorphine dose was observed. Diagnostic ultrasound may be useful as a non-invasive method of assessing subcutaneous nodule formation, to measure changes in affected tissue over time and after treatment.
\end{abstract}

Keywords: Parkinson's Disease, Panniculitis, Focal and Diffuse Tissue Changes, Dermal Thickness

\section{Introduction}

The drug apomorphine has been licensed for use in the United Kingdom since 1993 (Britannia Pharmaceuticals, Redhill, Surrey, UK). It is a dopamine agonist used for reducing unpleasant dyskinesias in people with advanced Parkinson's disease. ${ }^{1}$ Those who experience the greatest motor fluctuations, or debilitating, unpredictable 'on-off' periods, receive apomorphine subcutaneously via a continuous pump infusion normally during waking hours. The infusion site is usually the lower abdomen or upper thigh, ${ }^{1,2}$ and it is recommended that a new site for needle location is found each morning. ${ }^{3}$

The drug has common side-effects including nausea, confusion, sedation and cutaneous complications. ${ }^{1,2}$ Over time, the majority of people infusing apomorphine will develop painful hard nodules in and around the multiple injection sites. ${ }^{2,4,5}$ These painful nodules not only restrict patient choice of infusion site but may sometimes cause erratic absorption of the drug. ${ }^{1,4}$ The development of subcutaneous complications has been observed also in people with diabetes mellitus and in some sickle cell anaemia patients requiring regular intramuscular injections for pain control. ${ }^{6,7}$

Few studies have been conducted on the formation of apomorphine nodules and consequently, little is known about their aetiology or natural history. ${ }^{4,8}$ In this exploratory study, diagnostic ultrasound was used to characterise and catalogue the appearance of nodules in 12 patients. The study preceded an investigation into the effectiveness of a novel treatment strategy for such nodules, with ultrasonographic appearance being used as an outcome measure.

Correspondence: Hazel Edwards, School of Health \& Emergency Professions, University of Hertfordshire, College Lane, Hatfield, Herts, AL10 9AB, UK. h.m.edwards@herts.ac.uk

\section{Method}

Ethical approval for this exploratory study using ultrasound and for a proposed therapeutic trial, was obtained from the hospital trust ethics committee. Written informed consent was obtained from all participants. Twelve patients who used a continuous apomorphine pump infusion were recruited from a specialist Parkinson's disease clinic. Eight were female and four were male. Ages ranged from 47 to 75 years (mean age 64 years). The mean body mass index was 25.8 (range: $19 \cdot 1-$ 36.4). The number of years since Parkinson's disease was formally diagnosed ranged from 9 to 32 (mean 20 years). Apomorphine had been used by the participants for a mean average of four years (range: 3 months to 10 years) and the dose rate varied between 3.5 and $13.8 \mathrm{mgh}^{-1}$

The lower abdomen or upper thigh used for infusing was palpated manually to identify regions with the most profuse nodule formation. The affected tissue was photographed and then imaged by an experienced sonographer using a Sonosite MicroMaxx unit (Sonosite, Hitchin, UK) with colour Doppler and a 13-6 MHz linear array transducer.

The depth of field was kept constant at $4 \mathrm{~cm}$. The high resolution option with compound imaging was employed for maximum image quality. Images of the affected areas were acquired in both longitudinal and transverse planes. Control views of an area nearby which was not used for infusing were retained for comparison. Images were saved and stored for later analysis by the sonographer.

Soft copy images were scrutinised for both diffuse and focal subcutaneous and dermal changes. Normal subcutis has a uniform hypoechoic appearance and is traversed by thin septa (Fig. 1). ${ }^{9,10}$ Non-uniformity of the subcutis indicated the presence of nodules. Normal dermis is hyperechoic compared to the subcutis, with an even depth of $2 \mathrm{~mm}$ or less (Fig. 1). ${ }^{11}$ The boundary between normal dermis and subcutis is clearly delineated on ultrasound. In this study, the dermis was examined for thickness, uniformity, and boundary demarcation 


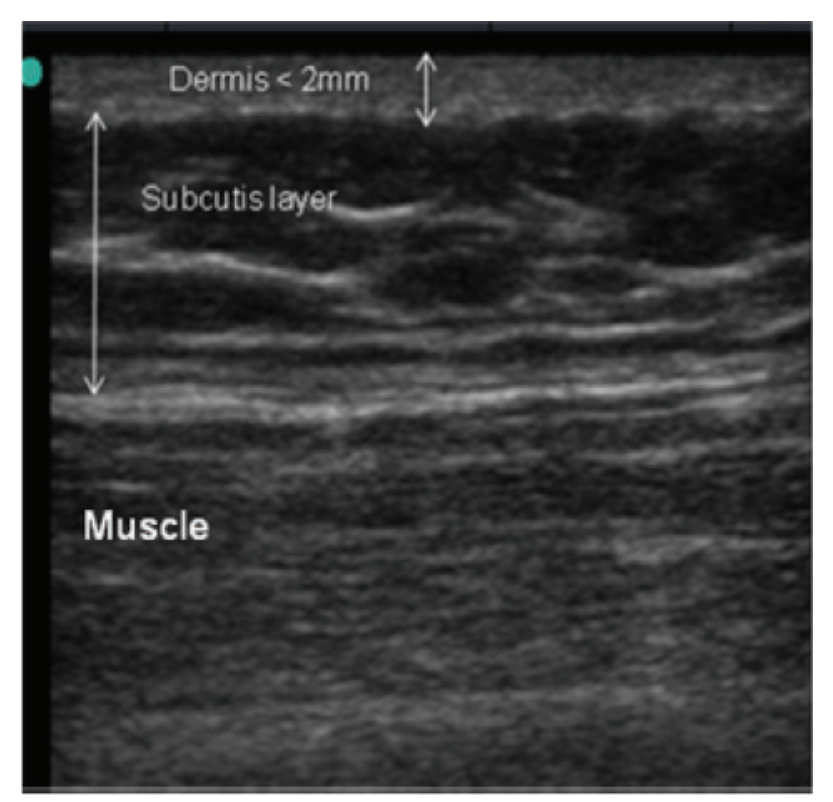

Figure 1. Normal abdominal dermis and subcutis.

from the subcutis. The subcutis was assessed for echogenicity compared to the control zones, and focal nodular areas within the subcutis were assessed for size, shape, edge definition, and echo-texture. Vascularity of the nodular areas was investigated using colour Doppler on a low velocity setting of $3 \mathrm{cms}^{-1}$

\section{Results}

All the control zones displayed normal dermal and subdermal sonographic appearances. In the areas used for infusion the dermis was thickened ( $3-8 \mathrm{~mm})$ in $42 \%(n=5)$ of the patients (Fig. 2), and the boundary between the dermis and subcutaneous layer was indistinct in 50\% $(n=6)$ (Fig. 3). When compared to control areas not used for infusion, the subcutaneous layer was diffusely hyperechoic in $42 \%(n=5)$ (Fig. 4a). In some cases these diffuse appearances correlated well with visible inflammatory changes on the skin surface (Fig. 4b).

From the cohort of twelve patients, 18 nodules were identified and analysed in detail. The majority were spherical

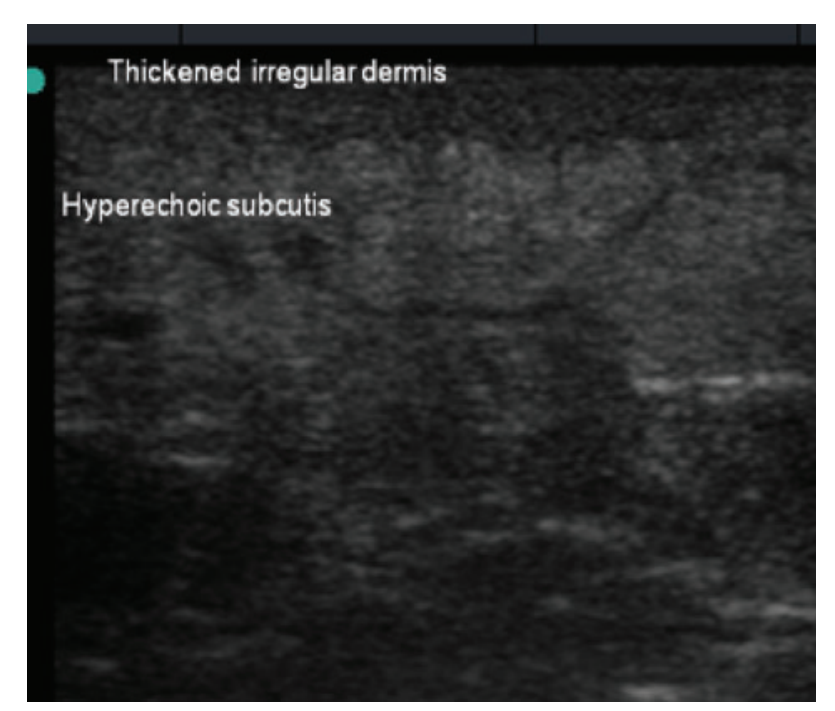

Figure 2. Thickened dermis and mildy hyperechoic subcutis.

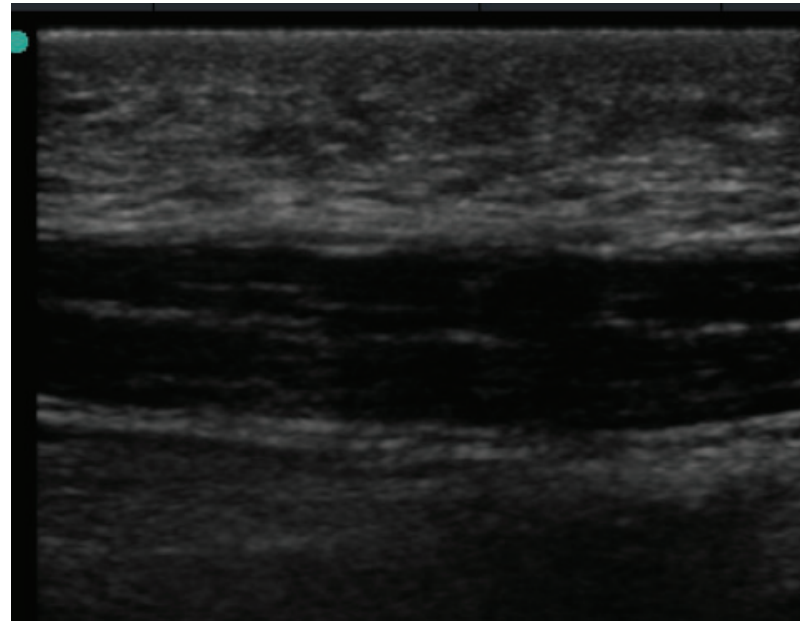

Figure 3. Indistinct dermal-subdermal boundary.

or fusiform in shape, ranging from 3 to $30 \mathrm{~mm}$ in diameter. Most nodules $(61 \%, n=11)$ had well-defined borders. Seven (39\%) were irregular or indistinct in outline. Four (22\%) nodules were hypoechoic compared to the surrounding subcutis (Fig. 5). Seven (39\%) were isoechoic or hyperechoic compared to the surrounding subcutis (Fig. 6a and b). Isoechoic nodules were identified by the presence of a hypoechoic halo. Seven nodules (39\%) were truly cystic in appearance (Fig. 7). One participant who had been using apomorphine in excess of 10 years demonstrated strongly shadowing linear foci in addition to having diffuse dermal and subdermal changes (Fig. 8). Multiple nodules within the same patient often had varying appearances. None of the 18 focal areas displayed any detectable vascularity on colour Doppler interrogation. There was no obvious correlation between patient demographics, drug dose and severity of nodule formation or other tissue changes.

\section{Discussion}

Ultrasound has been used to examine the skin and subcutaneous layer for over 30 years and is now the imaging modality of choice due to its ability to achieve high resolution images and accurate reproducible measurements., 12-14 Numerous studies have used ultrasound to described a range of superficial focal and diffuse skin appearances including tumour, lipoma, haematoma, lymphoma, abscess, cellulitis and panniculitis. , 10,13-16 $^{2}$

We used ultrasound to investigate subcutaneous appearances in people using apomorphine infusions and found that in every case the infusion sites had markedly abnormal sonographic appearances compared to control areas. Focal and/or diffuse subdermal changes were observed in all patients. Many had dermal thickening and a loss of definition of the dermis/subcutis boundary. Diffuse hyperechoic appearances and indistinct delineation between dermis and subcutis are consistent with cellulitis. ${ }^{9,14,17}$ These observations indicate that apomorphine infusion is associated with more extensive changes in tissue than has previously been reported.

A number of causal processes are possible. Tissue changes may be the consequence of inflammatory effects caused by repeated needle insertion in a relatively small area. ${ }^{8,18}$ Associated haemorrhage through repeated needle trauma can lead to vascular compromise of the capillaries, which also influences subcutaneous appearances. ${ }^{6,9,19}$ Although colour Doppler did not reveal the presence of fine vessels in this study, power Doppler might have. 

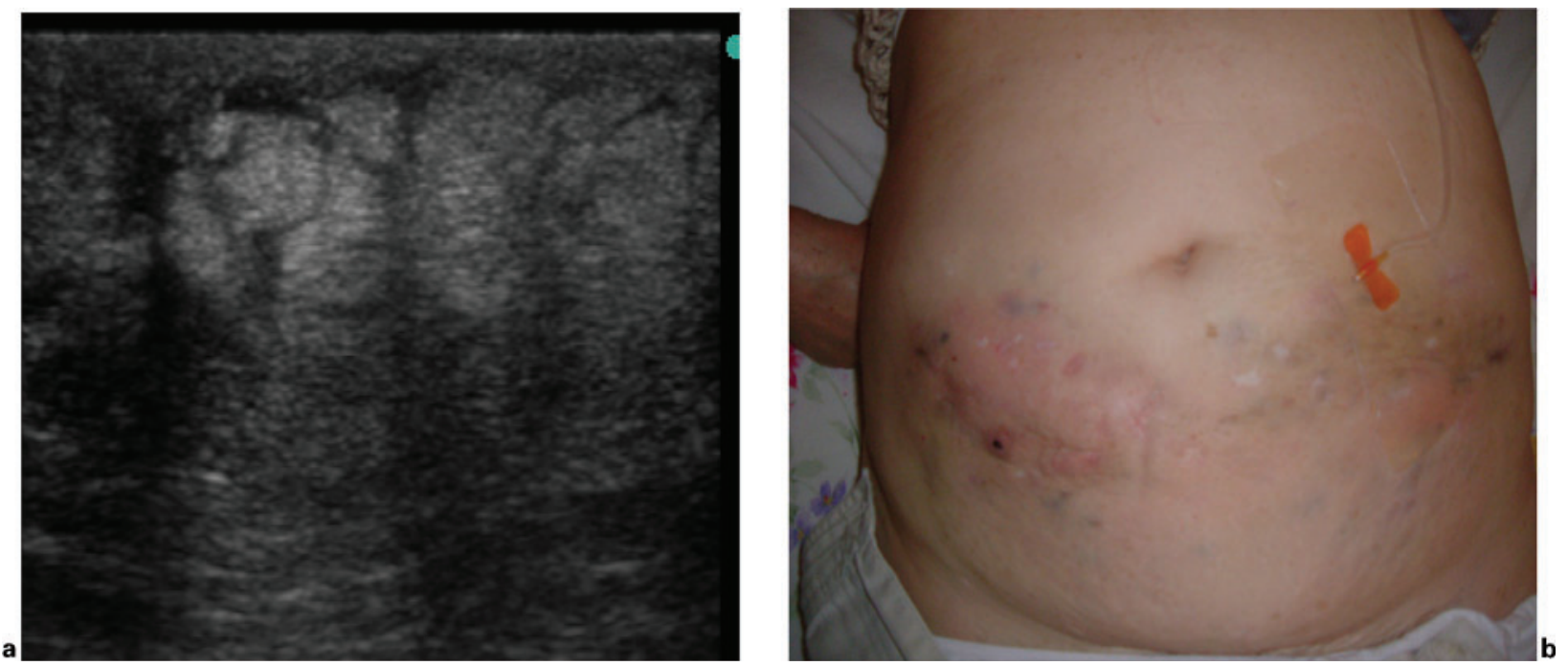

Figure 4. (a) Sonographic image of hyperechoic subcutis of the same patient. (b) Photograph of abdomen with apomorphine infusion in situ. Appearances suggest cellulitis and nodular formation.

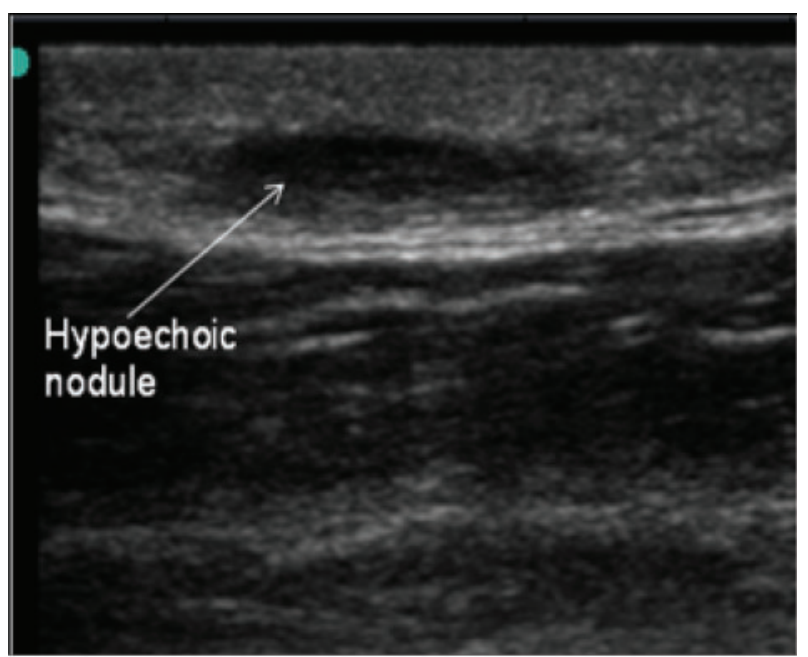

Figure 5. Hypoechoic nodule directly inferior to the dermis.

Traumatic panniculitis cannot be the only mechanism involved since nodule formation may occur after just one needle insertion in some patients. Nodule formation could also be triggered by subcutaneous tissue reacting to the drug itself or to its preservative. This is supported by evidence that nodule development is reduced when the drug is diluted with saline. ${ }^{1}$ Tissue changes may therefore be a combination of needle trauma and reaction to the drug compound.
Panniculitis is a complex condition involving inflammation of the subcutaneous layer beneath the dermis, ${ }^{20}$ which may result in fat necrosis. ${ }^{6}$ Different histopathological appearances are observed at different stages of panniculitis. ${ }^{20}$ Injecting drugs and performing needle biopsies is known to cause traumatic panniculitis and fat necrosis. ${ }^{9,19,21-23}$ The appearance of fat necrosis has been described most frequently in the breast where it can mimic cancer. ${ }^{19,21,24}$ Other studies report examples of fat necrosis in the thigh, ${ }^{6,9}$ and in the abdomen of a person with diabetes mellitus. ${ }^{.}$These nodules were also tender and resulted in erratic absorption of insulin.

Localised fat necrosis has varying sonographic appearances, ${ }^{9}$ depending on age, ${ }^{19,21,24}$ and presence of haemorrhage within the fat necrosis, ${ }^{6,19}$ although it is difficult to differentiate between the two processes using ultrasound alone. ${ }^{21,24}$ This may explain why a wide range of nodular appearances was seen in our study. Hyperechoic and hypoechoic foci in apomorphine users may represent not only nodules of different ages but also regions of fat necrosis mixed with varying degrees of haemorrhage. The truly cystic nodules observed may be in keeping with the evolution of old liquefied haematomata. ${ }^{25,26}$

Fibrotic changes in breast tissue cause acoustic shadowing on ultrasound. ${ }^{27}$ Strong shadowing seen in the subcutis of one participant in this study may also represent fibrotic tissue changes, especially in view of the length of time that person had been infusing apomorphine. Further studies on other longterm users are needed to investigate this suggestion.
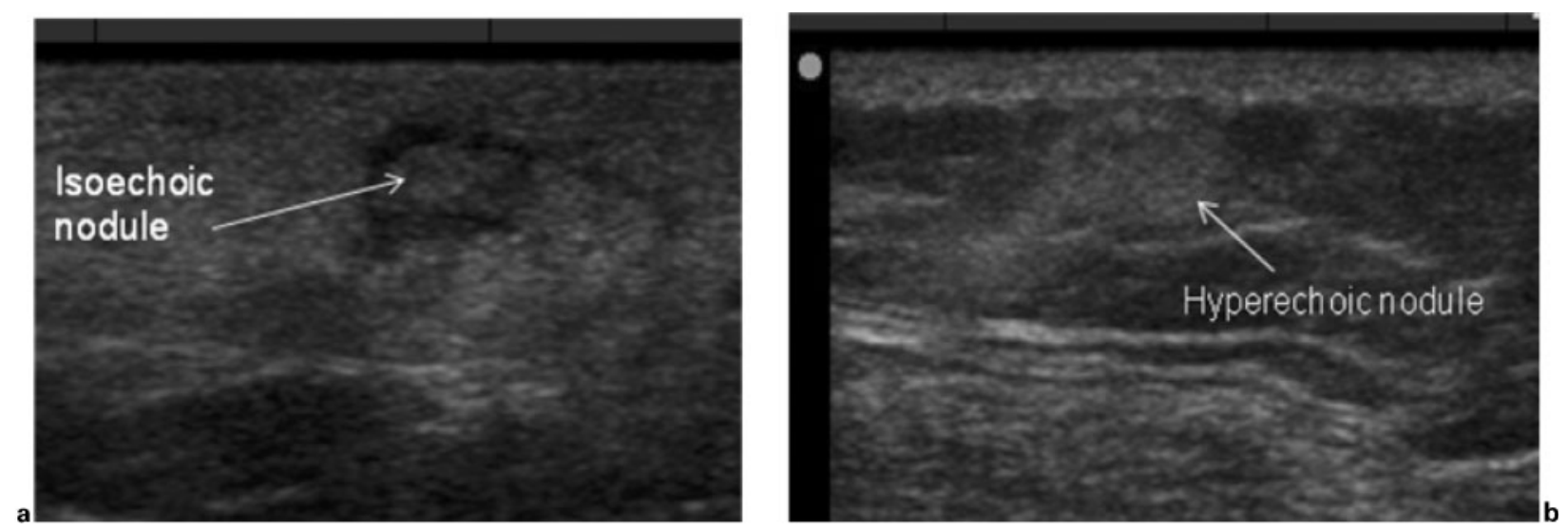

Figure 6. (a) Isoechoic nodule with hypoechoic border. (b) Hyperechoic nodule (normal dermis). 


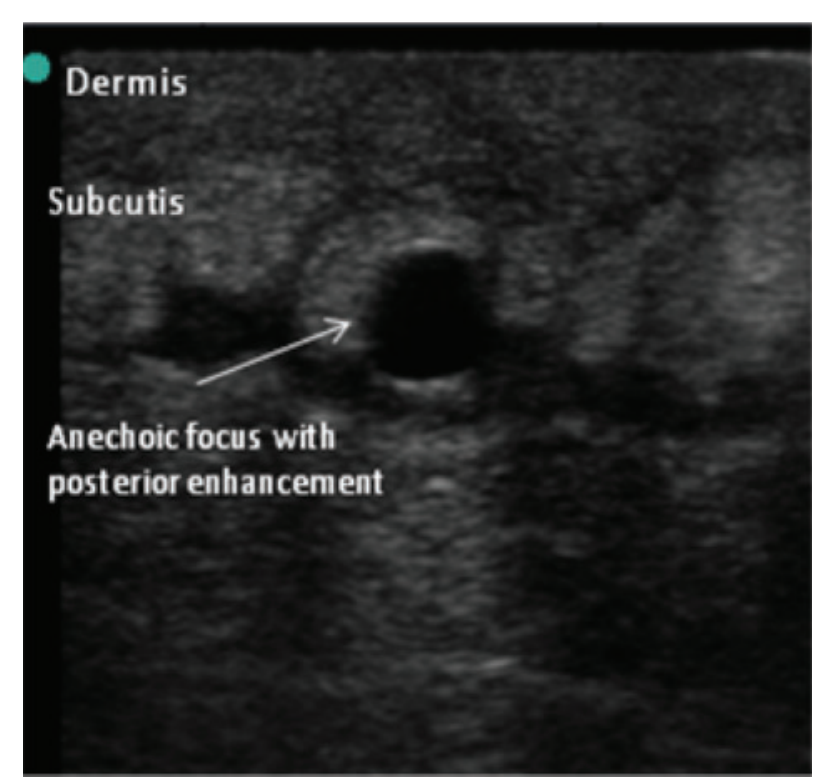

Figure 7. Anechoic or cystic focus (with thickened dermis and indistinct boundary).

Histological correlation with sonographic findings is necessary before any of these interpretations can be verified. Nevertheless our findings suggest that diagnostic ultrasound may provide a feasible outcome measure for tracking subcutaneous tissue changes. The data from this study will form the foundation for a follow-up study on response of these lesions to therapeutic ultrasound treatment.

Currently, there is limited evidence regarding prophylaxis or treatment of apomorphine nodules. Scrupulous hygiene when preparing to inject, massage of the affected areas, application of tea tree oil, silicone gel patches, and therapeutic ultrasound have all been suggested as measures to minimise nodule formation and hardness. ${ }^{1,2,4}$ A clearer understanding of nodule development and appearance is likely to help evaluate preventative measures and therapeutic pathways. While histology remains the gold standard in diagnosing and measuring subcutaneous tissue changes such as panniculitis, ultrasound has the advantage of being non-invasive, portable, reproducible and dynamic. Diagnostic ultrasound could have

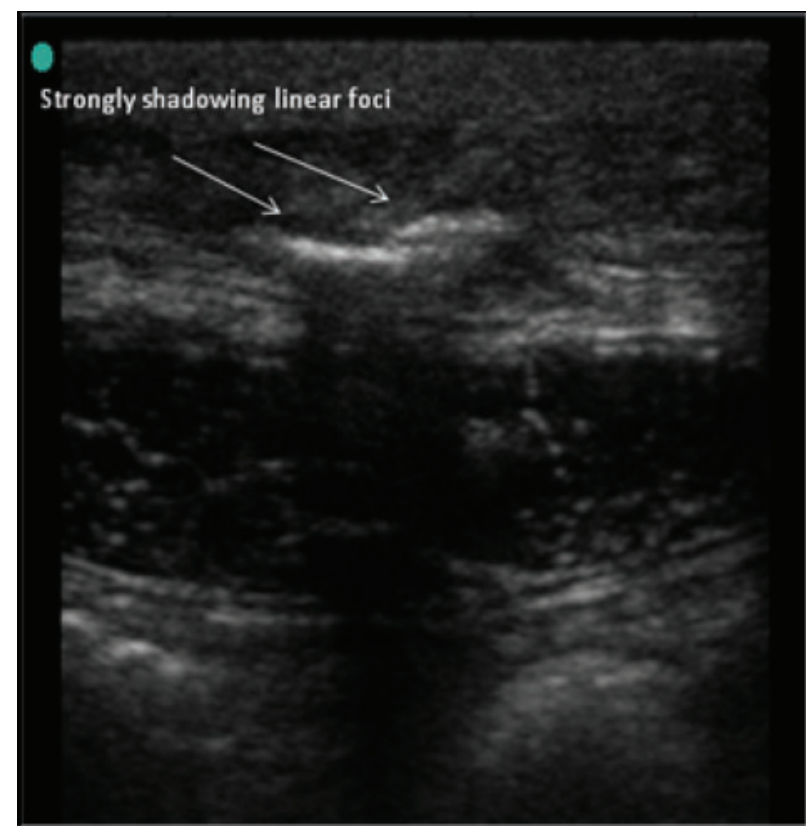

Figure 8. Strong acoustic shadowing from reflective foci (with thickened dermis). a role in the tracking of inflammatory changes and may prove to be a useful imaging modality for assessing the effectiveness of treatment strategies. ${ }^{28}$ Recommendations for further work include correlation of sonographic and histological findings, as well as tracking the evolution and resolution of nodules in a large cohort so that risk factors and prognostic indicators may be identified

\section{Conclusion}

In this study, there was a marked departure from normal in the sonographic appearances of skin and subcutaneous areas used for infusing apomorphine. The wide range of nodule appearances may be due to varying stages of inflammatory changes associated with the complex process of panniculitis. Diagnostic ultrasound may be helpful for tracking the evolution of apomorphine nodules and other changes in surrounding tissue.

\section{Acknowledgements}

The authors gratefully acknowledge the support of SonoSite UK Limited for supplying a MicroMaxx ultrasound system for this work. We also extend our thanks to the participants for taking part in this study, and to Professor Tim Watson, University of Hertfordshire, the research project lead.

\section{References}

1. Lees A, Turner K. Apomorphine for Parkinson's disease. Practical Neurology 2002;2:280-286

2. Bowron A. Practical considerations in the use of apomorphine injectable. Neurology 2004;62:S32-S36.

3. James CR, Quinn NP, Lees AJ, Swinn LA. Parkinson's disease and apomorphine. A guide for patients and those who care for them. University College London Hospitals NHS Foundation Trust, 2005

4. Manson A, Hanagasi $\mathrm{H}$, Turner $\mathrm{K}$, et al. Intravenous apomorphine therapy in Parkinson's disease. Clinical and pharmacokinetic observations. Brain 2001;124:331-340.

5. Pollak P, Benabid A, Limoson P, Jeanneau-Nicolle E. External and implanted pumps for apomorphine infusion in Parkinsonism Acta Neurochir Supplement Wien 1993;58:48-52.

6. Fernando R, Somers S, Edmonson R, Sidhu PS. Subcutaneous fat necrosis. Hypoechoic appearance on sonography. J Ultrasound Med 2003;22:1387-1390.

7. Wallymahmed M, Littler P, Clegg C, Haqqani MT, MacFarlane IA Nodules of fibrocollagenous scar tissue induced by subcutaneous insulin injections: a cause of poor diabetic control. Postgrad Med J 2004;80:732-733.

8. Acland K, Churchyard A, Fletcher C, Turner K, Lees A, Dowd PM Panniculitis in association with apomorphine infusion. $\mathrm{Br} \mathrm{J}$ Dermatol 1998;138:480-482

9. Sidhu P, Rich P. Sonographic detection and characterization of musculoskeletal and subcutaneous tissue abnormalities in sickle cell disease. Br J Radiol 1999;72:9-17.

10. Ulrich J, Voit, C. Ultrasound in dermatology. Part II. Ultrasound of regional lymph node basins and subcutaneous tumours. Eur J Dermatol 2001;11:73-79

11. Moore T, Lunt M, McManus B, Anderson ME, Herrick AL. Seventeen-point dermal ultrasound scoring system - a reliable measure of skin thickness in patients with systemic sclerosis. Rheumatology 2003;42:1559-1563.

12. Goldberg B. Ultrasonic evaluation of superficial masses. J Clin Ultrasound 1975;3:91-94. 
13. Nessi R, Betti R, Bencini P, Crosti C, Blanc M, Uslenghi C. Ultrasonography of nodular and infiltrative lesions of the skin and subcutaneous tissues. J Clin Ultrasound 1990;18:103-109.

14. Beggs I. Ultrasound of soft tissue masses. Imaging 2002; 14:202-208.

15. Loyer E, DuBrow R, David C, Coan JD, Eftekhari F. Imaging of superficial soft tissue infections: sonographic findings in cases of cellulitis and abscess. Am J Roentgenol 1996;166:149-152.

16. Wortsman X, Holm E, Wulf H, Jemec GB. Real-time spatial compound ultrasound imaging of skin. Skin Research \& Technology 2004;10:23-31.

17. Loyer E, Kaur H, David C, DuBrow R, Eftekhari FM. Importance of dynamic assessment of the soft tissues in the sonographic diagnosis of echogenic superficial abscesses. J Ultrasound Med 1995; 14:669-671.

18. van Laar T, van Hilten $\mathrm{B}$. The role of EDTA in provoking allergic reactions to subcutaneous infusion of apomorphine in patients with Parkinson's disease. J Neurol 1998;251:1370-1374

19. Soo M, Kornguth $P$, Hertzberg B. Fat necrosis in the breast: sonographic features. Radiology 1998;206:261-269.

20. Cascajo C, Borghi S, Weyers W. Panniculitis: Definition of terms and diagnostic strategy. Am J Dermatopathol 2000;22:530-549.
21. Bilgen G, Ustun E, Memis A. Fat necrosis of the breast: clinical, mammographic and sonographic features. Eur J Radiol 2001:39:92-99.

22. Förström L, Winkelmann R. Factitial panniculitis. Arch Dermato 1974;110:747-750.

23. Patterson J. Differential diagnosis of panniculitis. Adv Dermatol 1991;6:309-329

24. Chala $L$, de Barros $N$, de Camargo Moraes $P$, et al. Fat necrosis of the breast: mammographic, sonographic, computed tomography and magnetic resonance imaging findings. Curr Probl Diagn Radiol 2004;33:106-126.

25. Bates J. Abdominal Ultrasound, how why \& when. 2nd edn Edinburgh: Churchill Livingstone, 2004;84, 187.

26. Wilson D, McLardy-Smith P, Woodham C, MacLarnon JC. Diagnostic ultrasound in haemophilia. J Bone Joint Surg $\mathrm{Br}$ 1987;69B:103-107.

27. Baillie M, Mok P. Fat necrosis in the breast: review of the mammographic and ultrasound features, and a strategy for management. Australas Radiol 2004;48:288-295.

28. Chan V, Soans B, Mathers D. Ultrasound and magnetic resonance imaging features in a patient with eosinophilic fasciitis. Australas Radiol 2004;48:414-417 